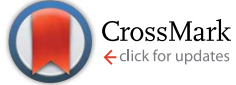

Cite this: J. Mater. Chem. A, 2017, 5, 6569

Received 30th January 2017

Accepted 6th March 2017

DOI: $10.1039 / c 7 t a 00967 d$

rsc.li/materials-a

\section{Enhanced pyroelectric and piezoelectric properties of PZT with aligned porosity for energy harvesting applications $\uparrow$}

\author{
Yan Zhang, ${ }^{a}$ Mengying Xie, ${ }^{a}$ James Roscow, ${ }^{a}$ Yinxiang Bao, ${ }^{b}$ Kechao Zhou, ${ }^{\text {b }}$ \\ Dou Zhang*b and Chris R. Bowen*a
}

\begin{abstract}
This paper demonstrates the significant benefits of exploiting highly aligned porosity in piezoelectric and pyroelectric materials for improved energy harvesting performance. Porous lead zirconate (PZT) ceramics with aligned pore channels and varying fractions of porosity were manufactured in a waterbased suspension using freeze-casting. The aligned porous PZT ceramics were characterized in detail for both piezoelectric and pyroelectric properties and their energy harvesting performance figures of merit were assessed parallel and perpendicular to the freezing direction. As a result of the introduction of porosity into the ceramic microstructure, high piezoelectric and pyroelectric harvesting figures of merits were achieved for porous freeze-cast PZT compared to dense PZT due to the reduced permittivity and volume specific heat capacity. Experimental results were compared to parallel and series analytical models with good agreement and the PZT with porosity aligned parallel to the freezing direction exhibited the highest piezoelectric and pyroelectric harvesting response; this was a result of the enhanced interconnectivity of the ferroelectric material along the poling direction and reduced fraction of unpoled material that leads to a higher polarization. A complete thermal energy harvesting system, composed of a parallel-aligned PZT harvester element and an AC/DC converter, was successfully demonstrated by charging a storage capacitor. The maximum energy density generated by the 60 vol\%

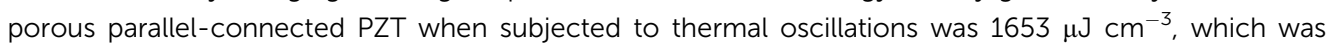
$374 \%$ higher than that of the dense PZT with an energy density of $446 \mu \mathrm{J} \mathrm{cm}^{-3}$. The results are beneficial for the design and manufacture of high performance porous pyroelectric and piezoelectric materials in devices for energy harvesting and sensor applications.
\end{abstract}

\section{Introduction}

Energy scavenging, or energy harvesting, which involves the conversion of ambient energy sources of energy such as mechanical vibrations, heat and light into useful electrical energy, continues to receive significant industrial and academic interest. This stems from the ability of harvesting systems to provide a route for the realization of autonomous and selfpowered low-power electronic devices. ${ }^{1-3}$ Among the wide variety of potential sources of ambient energy, the harvesting of energy from vibrations, movement, sound and heat is considered to be a highly promising energy harvesting technology. ${ }^{4-6}$

Mechanical waste energy that is generated by a vibrating structure or a moving object is a ubiquitous form of energy that

${ }^{a}$ Department of Mechanical Engineering, University of Bath, BA2 7AY, UK. E-mail: C.R. Bowen@bath.ac.uk

${ }^{b}$ State Key Laboratory of Powder Metallurgy, Central South University, 410083, China. E-mail:dzhang@csu.edu.cn

† Electronic supplementary information (ESI) available. See DOI: 10.1039/c7ta00967d can be harvested from our surroundings. ${ }^{7}$ The three common mechanisms for the conversion of vibrations into electricity include electromagnetic, electrostatic and piezoelectric techniques. ${ }^{\mathbf{8} 9}$ Piezoelectric materials have significant potential due to their high power density, and adaptability in terms of the variety of macro- and micro-scale fabrication methods. ${ }^{\mathbf{1 0 , 1 1}}$ In addition to mechanical energy, waste heat is a necessary byproduct of all thermodynamic cycles implemented in power, refrigeration, and heat pump processes ${ }^{\mathbf{1 2}}$ and recovering even a fraction of this energy has the potential for an economic and environmental impact. The conversion of heat directly into electricity can be achieved by thermoelectricity ${ }^{\mathbf{1 3}}$ or pyroelectricity. ${ }^{14}$ The advantages of pyroelectric materials, compared to thermoelectrics, is that they do not require bulky heat sinks to maintain a temperature gradient, and have the ability to operate with a high thermodynamic efficiency for converting temperature fluctuations into useable electrical power. ${ }^{15}$ Energy conversion by heating and cooling a pyroelectric material therefore offers a novel way to convert heat into electricity and the approach has attracted interest in application areas such as low-power electronics and battery-less wireless sensors. ${ }^{16}$ Since 
all pyroelectric materials are piezoelectric it is of significant interest to utilize piezoelectric and pyroelectric materials for energy harvesting applications with electrical properties that are readily adjustable and tailorable to suit the harvestable energy source and are also sufficiently robust to survive any applied mechanical loads and thermal strains.

In order to assess the properties of materials for energy harvesting, a variety of performance figures of merit containing combinations of physical properties have been developed to describe the ability of materials to generate energy for practical applications. For piezoelectric and pyroelectric energy harvesting applications, the following figures of merit have been widely used for the selection and design of materials: ${ }^{17-20}$

$$
\begin{gathered}
\mathrm{FoM}_{i j}=\frac{d_{i j}{ }^{2}}{\varepsilon_{0} \varepsilon_{33}{ }^{T}} \\
F_{\mathrm{E}}^{\prime}=\frac{p^{2}}{\varepsilon_{0} \varepsilon_{33}^{T} \times\left(C_{\mathrm{E}}\right)^{2}}
\end{gathered}
$$

where $\mathrm{FoM}_{i j}$ is a piezoelectric harvesting based figure of merit to assess the electrical energy density under a mechanical stress and $F_{\mathrm{E}}^{\prime}$ is a pyroelectric harvesting figure of merit indicating the energy density due to a thermal input. In eqn (1) and (2), $p$ is the pyroelectric coefficient, $\varepsilon_{33}^{T}$ is the relative permittivity (or dielectric constant), $\varepsilon_{0}$ is the permittivity of free space, $C_{\mathrm{E}}$ is the volume specific heat capacity and $d_{i j}$ is the piezoelectric charge coefficient, where $d_{33}$ and $d_{31}$ are the longitudinal and transverse piezoelectric charge coefficients respectively. These figures of merit take into account the piezoelectric, pyroelectric and thermal properties and therefore indicate which materials are likely to perform well in specific energy harvesting applications. Eqn (1) and (2) indicate that the important requirements for improved performance are low relative permittivity, low specific heat capacity and high piezoelectric and pyroelectric coefficients.

The two figures of merit above indicate that a porous structure whose permittivity is reduced due to the introduction of low permittivity pores would have beneficial consequences for piezo- and pyro-electric energy harvesting applications; however, it should be noted that the benefit is only achieved if the piezoelectric or pyroelectric coefficients are not reduced significantly by the presence of the pores. In general, it has been considered that porous piezoelectric and pyroelectric materials can be considered as a porosity (air)-ceramic matrix composite. ${ }^{21,22}$ It is well known that the properties of a ferroelectric composite depend on the depolarization factor, the connectivity of the phases and the permittivity ratio the two phases. ${ }^{23}$

\subsection{Porous piezoelectric composites with 2-2 connectivity}

Laminated 2-2 connectivity based piezoelectric and pyroelectric composites are of interest due to their simple architecture and superior actuation and sensing abilities. ${ }^{24-26}$ In the ' $2-2$ ' system the first number describes the connectivity of the active PZT ceramic phase and the second refers to the passive matrix phase (in this case the pore volume). It has been demonstrated in our previous research ${ }^{27}$ that PZT formed via ice-templating with highly aligned porosity exhibits a characteristic 2-2 connectivity along the freezing and poling directions. These materials exhibited a mechanical strength $\sim 300 \%$ higher than conventional porous structures with randomly distributed porosity, and the pyroelectric figure of merits are $140-360 \%$ times higher than the dense counterpart. Due to the unidirectional freezing nature of the ice-templating method, the resulting microstructure is highly oriented and strongly influences the corresponding physical, chemical and mechanical properties of the composite; these properties will be different in the directions that are parallel and perpendicular to the freezing direction, but are yet to be reported in detail for ferroelectric materials.

In this work, freeze-casting, also termed ice-templating, was utilized to fabricate porous PZT ceramics with tailored and aligned porosity to produce high performance materials for piezoelectric and pyroelectric energy harvesting applications. The piezoelectric and pyroelectric parameters and figures of merit for the energy harvesting applications of the porous PZT ceramics both parallel and perpendicular to the freezing direction have been investigated in detail. Based on the properties of these highly aligned materials, the porous composites are then utilized in an energy harvester and are shown to generate more energy compared to the dense material. The work demonstrates that tailored porosity can generate improved materials for piezoelectric and pyroelectric energy harvesting devices.

\section{Experimental}

Commercial lead zirconate titanate powder (PZT-51, Zibo Yuhai Electronic Ceramic Co., Ltd, Zibo, China) with a mean particle size of $\sim 3 \mu \mathrm{m}$ was ball-milled for $48 \mathrm{~h}$ using a planetary ball mill to reduce the particle size $d_{50}$ to $\sim 0.5 \mu \mathrm{m}$. Deionized water, polyvinyl alcohol (PVA, 420, Kuraray Co. Ltd, Japan) and ammonium polyacrylate (HydroDisper A160, Shenzhen Highrun Chemical Industry Co. Ltd, P. R. China) were used as the freezing vehicle, binder and dispersant, respectively. PZT suspensions with different solid loadings consisting of PZT powders, $1 \mathrm{wt} \%$ dispersant and $1 \mathrm{wt} \%$ PVA binder were ballmilled for $24 \mathrm{~h}$ in zirconia media and de-aired by stirring in a vacuum desiccator until complete removal of air bubbles. In the freeze-casting process, the freezing operation is undertaken by a customized double-sided setup as described previously. ${ }^{28,29}$ The PZT suspensions were poured into a cylindrical polydimethylsiloxane (PDMS) mold ( $\phi: 10 \mathrm{~mm}, H: 15 \mathrm{~mm}$ ), which was then transported to a copper cold finger placed in a liquid nitrogen container. After solidification of the mixture was complete, the samples were freeze-dried for $24 \mathrm{~h}$ via ice sublimation in a vacuum chamber ( $\sim 10 \mathrm{~Pa}$ ) of a freeze-drier (FD-1A50, Beijing Boyikang Medical Equipment Co., Beijing). The porous green bodies were placed in a furnace and heated in air with a heating rate of $1{ }^{\circ} \mathrm{C} \mathrm{min}{ }^{-1}$ at $500{ }^{\circ} \mathrm{C}$ for $2 \mathrm{~h}$ to burn out the organic additives and sintered at $1200{ }^{\circ} \mathrm{C}$ for $2 \mathrm{~h}$ under a PbO-rich atmosphere, then finally allowed to cool down naturally in a closed furnace. 
During the freeze-casting process, dense and cellular zones are formed in the samples at the beginning of the freezing cycle, as shown in Fig. 1(A). This region has a total thickness of less than $250 \mu \mathrm{m}$ (ref. 30) and is removed by cutting the lower 0.5 $\mathrm{mm}$ of material via a low-speed precision diamond sectioning saw machine (Buehler, IsoMet LS). The freeze-cast porous samples of the same porosity (Fig. 1(B)) were further cut both parallel (Fig. 1(C)) and perpendicular (Fig. 1(D)) to the freezing direction to assess their properties in different orientations with almost the same volume. The thickness, $c$, of both types of porous samples was $\sim 1.0 \mathrm{~mm}$. Samples cut parallel to the freezing direction had a length $a=14.63 \pm 0.43 \mathrm{~mm}$ and width $b=5 \mathrm{~mm}$. Samples cut perpendicular to the freezing direction had a diameter, $\phi=9.65 \pm 0.14 \mathrm{~mm}$.

The microstructures of the sintered samples, such as their pore structures and the structure of the PZT walls, were characterized using scanning electron microscopy (SEM, JSM6480LV, JEOL Techniques, Tokyo, Japan). The apparent porosity of the composites was derived from density data obtained by the Archimedes method. ${ }^{31}$ The compressive strength of the sintered sample (diameter $\sim 9.5 \mathrm{~mm}$ and height $\sim 13$ $\mathrm{mm}$ ) was measured with a crosshead speed of $0.2 \mathrm{~mm} \mathrm{~min}^{-1}$ using an Electronic Universal Testing Machine (KD11-2, Shenzhen KEJALI Technology Co. Ltd, China). An average of five measurements was taken for each sample. The porosity of the porous ceramic was calculated using eqn (3):

$$
\text { Porosity }=1-\frac{\rho_{\text {porous PZT }}}{\rho_{\text {bulk }}} \times 100 \%
$$

where $\rho_{\text {porous PZT }}$ is the density of the porous sample and $\rho_{\text {bulk }}$ is the density of the bulk PZT.

Before electrical properties were measured, corona poling was conducted on the sintered dense and porous samples at $120{ }^{\circ} \mathrm{C}$ by applying a potential difference of $14 \mathrm{kV}$ to a point source above the sample for $15 \mathrm{~min}$, and samples were then aged for $24 \mathrm{~h}$ before testing. The longitudinal piezoelectric strain coefficient $\left(d_{33}\right)$ and the transverse piezoelectric strain coefficient $\left(d_{31}\right)$ were measured using a Berlincourt Piezometer (PM25, Take Control, UK). Measurements of the relative permittivity $\left(\varepsilon_{33}^{T}\right)$ of the sintered materials were carried out from

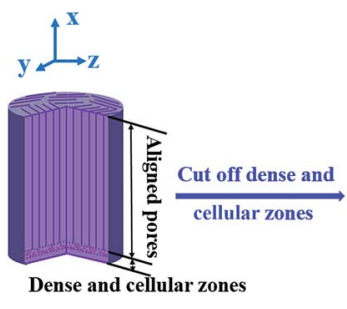

(A)

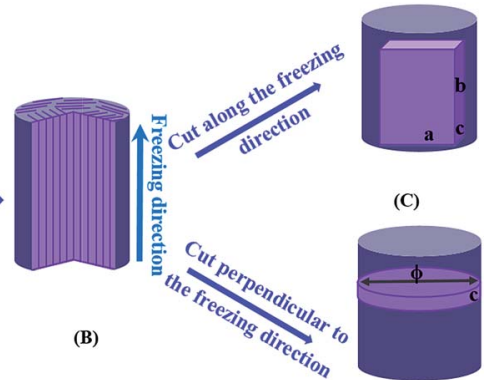

(D)
Fig. 1 Schematic of the preparation of the freeze-cast samples. (A) Sintered PZT ceramic, (B) aligned porous PZT sample after removing the dense and cellular zones, (C) cutting along the freezing direction and (D) perpendicular to the freezing direction.
$1 \mathrm{~Hz}$ to $1 \mathrm{MHz}$ at room temperature $\left(\sim 30^{\circ} \mathrm{C}\right)$ using an impedance analyzer (Solartron 1260, Hampshire, UK). Ferroelectric properties, such as the maximum polarization, remnant polarization and coercive field, were measured using a Radiant RT66B-HVi Ferroelectric Test system on unpoled materials. The specific heat capacity $\left(C_{\mathrm{p}}\right)$ of PZT composites with different porosities was measured from 20 to $100{ }^{\circ} \mathrm{C}$ by a MicrosC multicell calorimeter from Setaram, with the Calisto program to collect and process the data. The density of the solid PZT, $\rho$, is $7.6 \mathrm{~g} \mathrm{~cm}^{-3}$ (according to the data sheet), therefore the volume specific heat, $C_{\mathrm{V}}$, can be defined as:

$$
C_{\mathrm{V}}=\rho C_{\mathrm{p}}
$$

The pyroelectric short circuit current $\left(I_{\mathrm{P}}\right)$ and pyroelectric open circuit voltage (V) were measured using an electrometer (Model 6517B, Keithley Instruments, Cleveland, OH), and the pyroelectric coefficient was determined by the Byer-Roundy method $^{32}$ and derived from:

$$
I_{\mathrm{P}}=p A \frac{\mathrm{d} T}{\mathrm{~d} t}
$$

To demonstrate the effectiveness of the porous materials a thermal energy harvesting demonstrator was constructed, with a $1 \mu \mathrm{F}$ charging capacitor used as an electrical storage element when the energy harvester was subjected to cyclic temperature fluctuations at $1.6{ }^{\circ} \mathrm{C} \mathrm{s}^{-1}$. The temperature of the sample was continuously monitored using a K-type thermocouple with a response time of $0.5 \mathrm{~s}$. A charging curve of the storage capacitor was measured for the range of materials, resulting in a maximum voltage on the storage capacitor without any electrical load.

\section{Results and discussion}

\subsection{Microstructure and mechanical properties}

As an example of a typical microstructure, Fig. 2(A)-(D) show SEM images of the microstructures of sintered freeze-cast PZT ceramics in directions perpendicular (Fig. 2(A)) and parallel (Fig. 2(B)) to the unidirectional freezing direction for a composite with a porosity level of $40 \mathrm{vol} \%$. The presence of highly aligned and homogeneous lamellar pore channels with a pore width of 10-13 $\mu \mathrm{m}$ and a dense ceramic wall thickness of 5-8 $\mu \mathrm{m}$ can be observed in both directions, similar to our previous reports. ${ }^{28,29}$ The porous PZT ceramic parallel to the freezing direction exhibits a higher degree of parallelism compared to the perpendicular direction; compare Fig. 2(A) and (B). In contrast to conventional isotropic porous structures with uniformly distributed porosity, ${ }^{33}$ a high fraction of ceramic links in the pore volume of the freeze-cast porous PZT is observed in both directions. These ceramic links are located in the adjacent lamellar walls of the long-range ordered pore channels, as shown by the red arrows of Fig. 2(C) and (D) which show higher magnification images of the microstructure. These ceramic bridges have been observed in a wide variety of freezecast porous ceramics which employ water-based suspensions, ${ }^{30}$ 

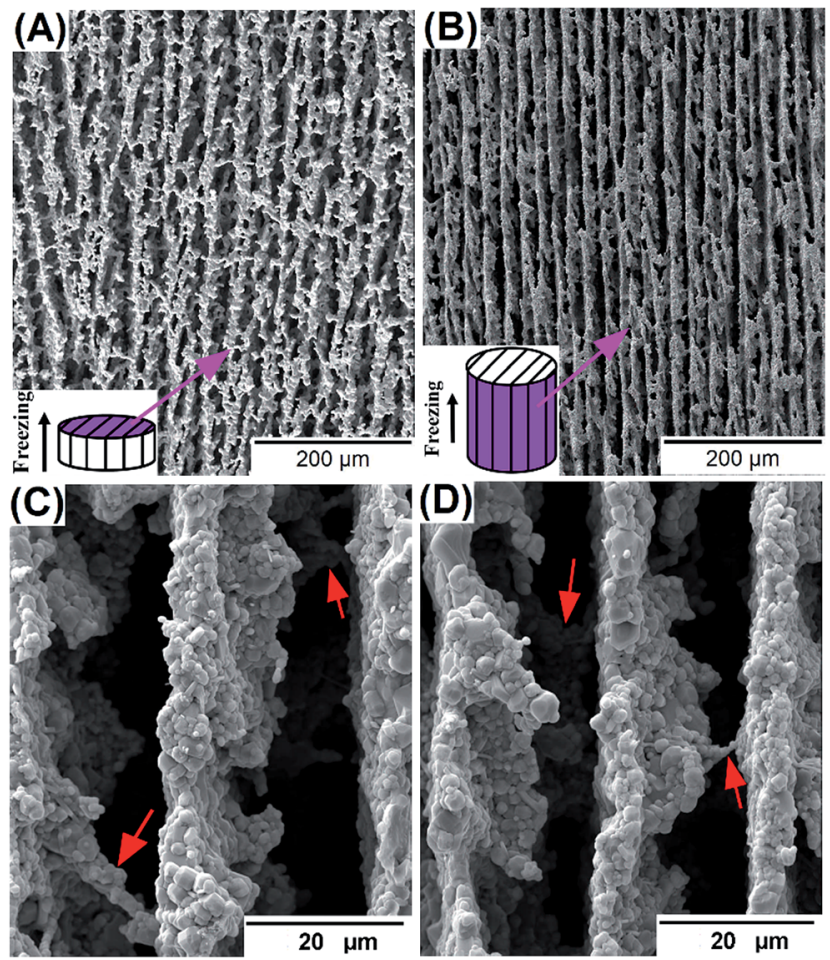

Fig. 2 SEM images of the (A) parallel-connected (perpendicular to the freezing direction) and (B) series-connected (along the freezing direction) porous PZT at a porosity of 40 vol\% with high magnification images in (C) and (D), respectively.

and for a variety of porosities ranging from 25 to $45 \mathrm{vol} \%$ in the porous PZT ceramics in our previous work. ${ }^{27}$ Under unidirectional freezing conditions, the growth velocity of the ice front along the $x$ - or $y$-axis in Fig. 1 was $10^{2}$ to $10^{3}$ times faster than in the direction perpendicular to the freezing direction (the $z$-axis in Fig. 1). ${ }^{30}$ This results in aligned lamellar pore channels and dendritic ceramic bridges that are a replica of the orientated growth of the ice crystals.

The compressive strengths of PZT ceramics with the porosities ranging from 20 to $60 \mathrm{vol} \%$ in both the parallel and perpendicular directions to the unidirectional freezing direction have been shown in Fig. S1. $\dagger$ Compared with the conventional porous PZT ceramics with uniformly distributed porosity, ${ }^{27}$ the PZT with pores perpendicular to the freezing direction exhibited a compressive strength of $320-580 \%$ higher than that of the conventional porous PZT for porosities ranging from 20 to $60 \mathrm{vol} \%$, see Fig. S1. $\dagger$ The strength along the freezing direction was $\sim 200 \%$ higher than that of the uniformly distributed porous PZT. This high strength results from the unidirectional lamellar support and the strong bonding originating from the ceramic bridges between the lamellar ceramic walls, as seen in Fig. 2 .

Fig. 3 shows a schematic diagram of the poling direction applied to the freeze-cast porous PZT that were cut both parallel (as in Fig. 1(C)) and perpendicular (as in Fig. 1(D)) to the freezing direction. Both types of PZT were explored to examine the influence of the pore orientation on the poling behaviour and electrical properties, as shown in Fig. 3(A) and (C). To

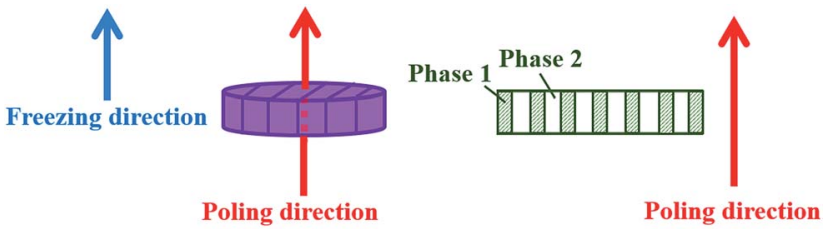

(A)

(C)

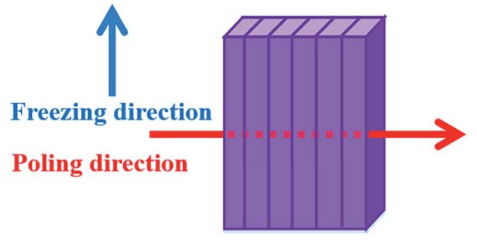

(B)

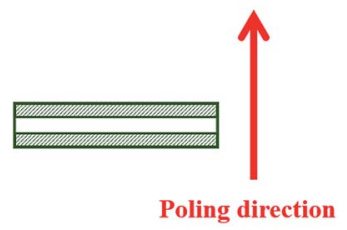

(D)
Fig. 3 Schematic diagram of the poling processes conducted on (A) parallel-connected and (C) series-connected porous PZT, which can be considered analogous to the classical (B) parallel and (D) series models, respectively.

model the influence of the composite microstructure, the highly aligned pores can be considered analogous to the classical parallel-connected (Fig. 3(B)) and series-connected (Fig. 3(D)) models used in estimating the piezoelectric and pyroelectric effects of diphasic composites, respectively. ${ }^{34}$

\subsection{Polarization-field $(\boldsymbol{P}-\boldsymbol{E})$ loops}

Fig. 4(A)-(D) show the $P-E$ hysteresis loops of the parallelconnected and series-connected freeze-cast PZT ceramics at different maximum electric fields and porosities, respectively. Fig. 4(A) and (C) show, as an example, the development of the $P$ $E$ loops with increasing maximum applied field for a $20 \mathrm{vol} \%$ porous ceramic. The $P-E$ loops become more square-shaped as the maximum applied field increases since the remnant polarization $\left(P_{\mathrm{r}}\right)$ and coercive field $\left(E_{\mathrm{c}}\right)$ increase. The $E_{\mathrm{c}}$ increases rapidly at maximum fields between 9 and $12 \mathrm{kV} \mathrm{cm}^{-1}$ due to increased domain switching for the parallel-connected PZT and between 9 and $15 \mathrm{kV} \mathrm{cm}^{-1}$ for the series-connected PZT.

The increase in $E_{\mathrm{c}}$ with maximum field then slows down substantially at higher applied fields due to saturation (13-16 $\mathrm{kV} \mathrm{cm}{ }^{-1}$ for parallel-connected, $18-24 \mathrm{kV} \mathrm{cm}{ }^{-1}$ for seriesconnected). Saturated and rectangular loops of the parallelconnected and series-connected freeze-cast PZT ceramics were achieved at $16 \mathrm{kV} \mathrm{cm}^{-1}$ and $24 \mathrm{kV} \mathrm{cm}^{-1}$ respectively; higher electric fields than those presented here could not be applied due to dielectric breakdown.

Fig. 4(B) and (D) show the parallel-connected and seriesconnected porous PZT ceramics with various porosities (20-60 vol\%), respectively. All materials exhibited well-developed and almost symmetric hysteresis loops. The $P_{\mathrm{r}}$ of the parallelconnected PZT decreased gradually from 16.5 to $6.4 \mu \mathrm{C} \mathrm{cm}^{-2}$, and the $E_{\mathrm{c}}$ monotonously increased from 7.7 to $9.1 \mathrm{kV} \mathrm{cm}^{-1}$ as the porosity fraction increased at a maximum electric field of 16 $\mathrm{kV} \mathrm{cm}^{-1}$. The $P_{\mathrm{r}}$ of the series-connected PZT decreased from 11.3 to $3.5 \mu \mathrm{C} \mathrm{cm}^{-2}$, and the $E_{\mathrm{c}}$ increased from 8.9 to $10.3 \mathrm{kV}$ $\mathrm{cm}^{-1}$ with increasing porosity at a maximum field of $24 \mathrm{kV}$ 

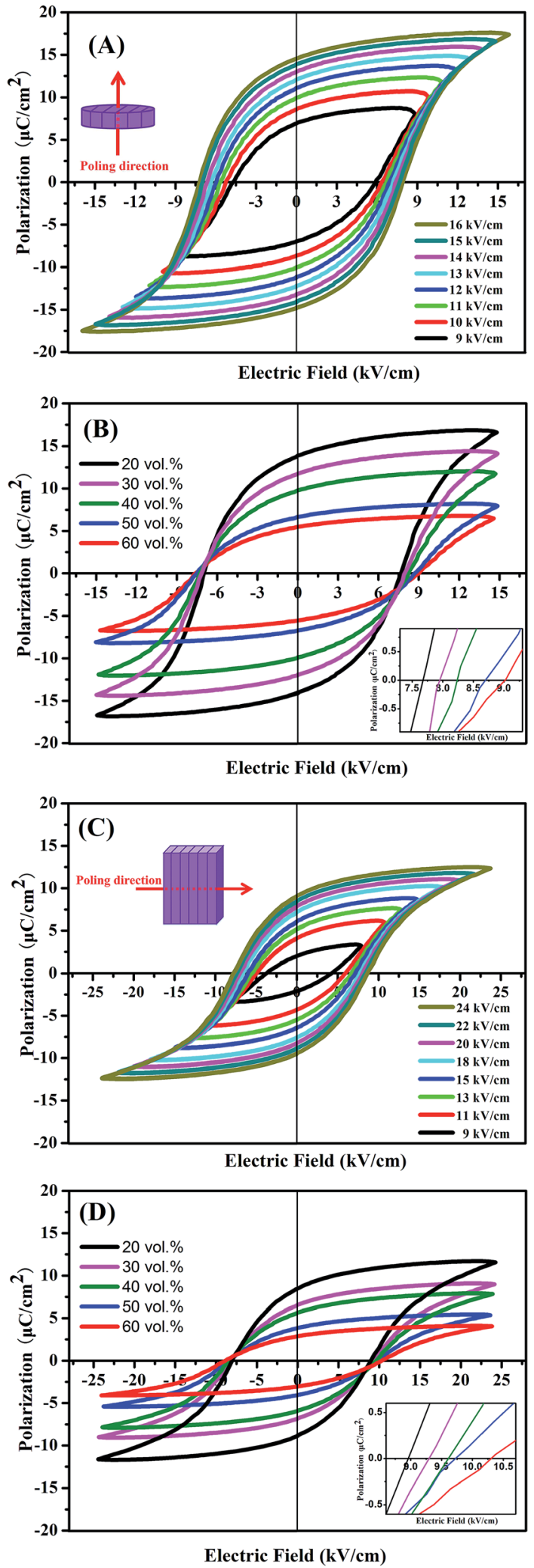

Fig. $4 P-E$ hysteresis loops of the parallel-connected and seriesconnected freeze-cast PZT. (A) Parallel-connected and (C) seriesconnected PZT with the porosity of 20 vol\% and different electric fields, (B) parallel-connected and (D) series-connected PZT with the electric field of $16 \mathrm{kV} \mathrm{cm}^{-1}$ and $24 \mathrm{kV} \mathrm{cm}^{-1}$ respectively with different porosities. Insets in (B) and (D) are the coercive fields of the porous freeze-cast PZTs. $\mathrm{cm}^{-1}$. Fig. $\mathrm{S} 2 \uparrow$ also shows for comparison the dense PZT with a $P_{\mathrm{r}}$ of $35.0 \mu \mathrm{C} \mathrm{cm} \mathrm{cm}^{-2}$ and coercive field of $8.7 \mathrm{kV} \mathrm{cm}^{-1}$.

For both types of porous PZT, the remnant polarization decreased gradually when the porosity increased from 20 to 30 vol\%, followed by a more rapid decrease when the porosity was higher than 40 vol\%. Increasing the porosity reduced the remnant polarisation since the porous PZT ceramics have proportionally reduced active material and ferroelectric domains compared with dense PZT, resulting in a lower polarisation. It should be noted that the parallel-connected PZT exhibited a more square $P-E$ loop, higher polarisation and lower coercive field compared to the series-connected PZT for the same porosity, e.g. at $40 \mathrm{vol} \% P_{\mathrm{r}}$ is $16.5 \mu \mathrm{C} \mathrm{cm}^{-2}$ and $E_{\mathrm{c}}$ is $8.25 \mathrm{kV}$ $\mathrm{cm}^{-1}$ for the parallel-connected PZT and is $11.3 \mu \mathrm{C} \mathrm{cm}^{-2}$ and $9.6 \mathrm{kV} \mathrm{cm}{ }^{-1}$ for the series-connected PZT. This is due to the improved interconnectivity of the PZT material along the polarisation direction for the parallel-connected PZT, see Fig. 3.

While the decrease in remnant polarisation with increasing porosity is simply due to the reduction in polarisable material, the increase in coercive field with an increase in porosity is less obvious. The porous ceramics are effectively composites with a high permittivity ceramic phase and a low permittivity pore phase (air). Such a structure strongly influences the distribution of the electric field throughout the material microstructure during the poling process. It has been shown that for porous ferroelectric ceramics, the local electric field in the ceramic phase near the pore is much lower than the applied field. This inhomogeneous field distribution results in the existence of unpoled areas along the poling direction. ${ }^{35}$ Consequently, the parallel-connected PZT exhibit less unpoled regions and easier domain switching and polarisation compared to the seriesconnected materials, leading to higher polarisation and lower coercive field.

A Finite Element Modelling approach has been used to investigate the effect of porosity in the freeze-cast microstructure on the electric field distribution in the PZT during the poling process, ${ }^{36}$ and to account for the increase in coercive field with increasing porosity (see Fig. 3(B) and (D)). Previous work $^{36}$ has demonstrated the ability of such models to predict the behaviour of porous PZT, although they have focused on uniformly distributed porosity within a ferroelectric material, i.e. systems with 3-0 and 3-3 connectivity. This approach has been adapted in this work to investigate the 2-2 connectivity structures achieved using the freeze-casting process. A three dimensional cubic mesh of 29791 (i.e. $31^{3}$ ) elements was split into an idealised 2-2 structure where alternate planes were assigned the properties of either unpoled PZT $\left(\varepsilon_{\mathrm{r}}=1585^{36}\right)$ or air $\left(\varepsilon_{\mathrm{r}}=1\right)$. In reality, the freeze-casting process does not produce ideal 2-2 structures due to ceramic links, as seen in Fig. 2(C) and (D), and therefore a further step was incorporated in which randomly selected elements in the pore channels were reassigned the properties of PZT and randomly selected elements in the PZT channels reassigned the properties of air. To create geometries of varying porosity $(20,30,40$ and 50 vol\%), the ratio of PZT channel width to pore channel width was varied. To model the electric field distribution in the dense material ( $96 \%$ of theoretical density), $4 \%$ of elements were 
randomly assigned the properties of air with the remaining elements assigned the properties of unpoled PZT. An electric field was applied across the structure parallel to the lamellar channel direction and the local electric field in each element was recorded.

Fig. 5 shows the electric field distribution for dense PZT (4 vol\% porosity) and ceramics with increasing porosity levels. A high electric field concentration is observed within the low permittivity pore volume, with regions of low electric field observed in the immediate vicinity of the pore parallel to the direction of the applied field, which in a ferroelectric material may lead to incomplete poling in these regions, as shown in Fig. 5. Meanwhile, a lower field is observed in the ceramic regions, compared to the lower permittivity pore channels. A higher external electric field must therefore be applied to switch domains in the ceramic regions and hence the coercive field increases with increasing porosity, as in Fig. 4(D). This phenomenon can be described by an adaptation of Gauss' law where the electric field, $E_{\mathrm{f}}$, is related to the relative permittivity by:

$$
E_{\mathrm{f}}=\frac{q}{A \varepsilon_{\mathrm{r}} \varepsilon_{0}}
$$

where $q$ is the charge, $A$ is the area and $\varepsilon_{0}$ is the permittivity of free space. In the dense ceramic, the electric field is relatively homogenous (see Fig. 5). However, in the porous structures the concentration of field in the low-permittivity pores results in the electric field no longer having a continuous and linear path. As the porosity level increases it can be seen in Fig. 5 that the electric field in the ceramic moves to lower magnitudes and is distributed over a wider distribution of electric fields. This results in an increase in coercive field with increasing porosity (as in Fig. 4(B) and (D)) since a larger external electric field must be applied to achieve domain switching.

\subsection{Piezoelectric and dielectric properties}

Fig. 6(A) and (B) show the longitudinal $\left(d_{33}\right)$ and transverse $\left(d_{31}\right)$ piezoelectric charge coefficients of the series-connected and the parallel-connected freeze-cast PZT ceramics, along with the dense material. As shown in Fig. 7, the piezoelectric $d_{33}$ and $d_{31}$

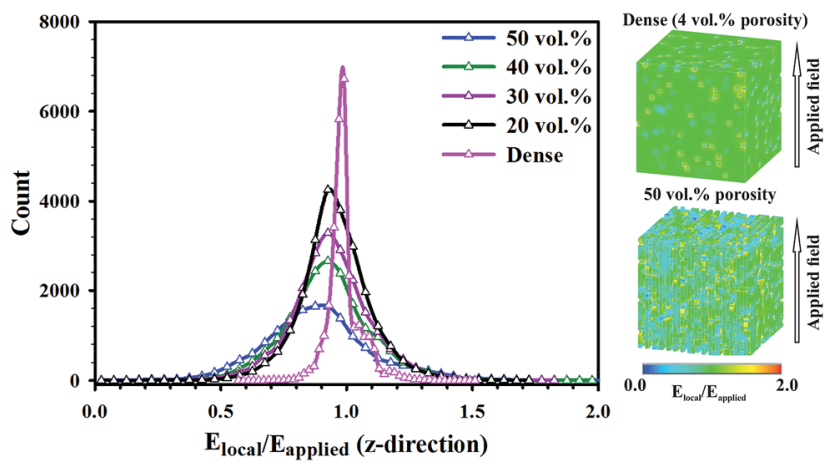

Fig. 5 Model of electric field distribution in porous PZT and for dense ( 4 vol\% porosity) to 50 vol\% obtained by the Finite Element Modelling approach.
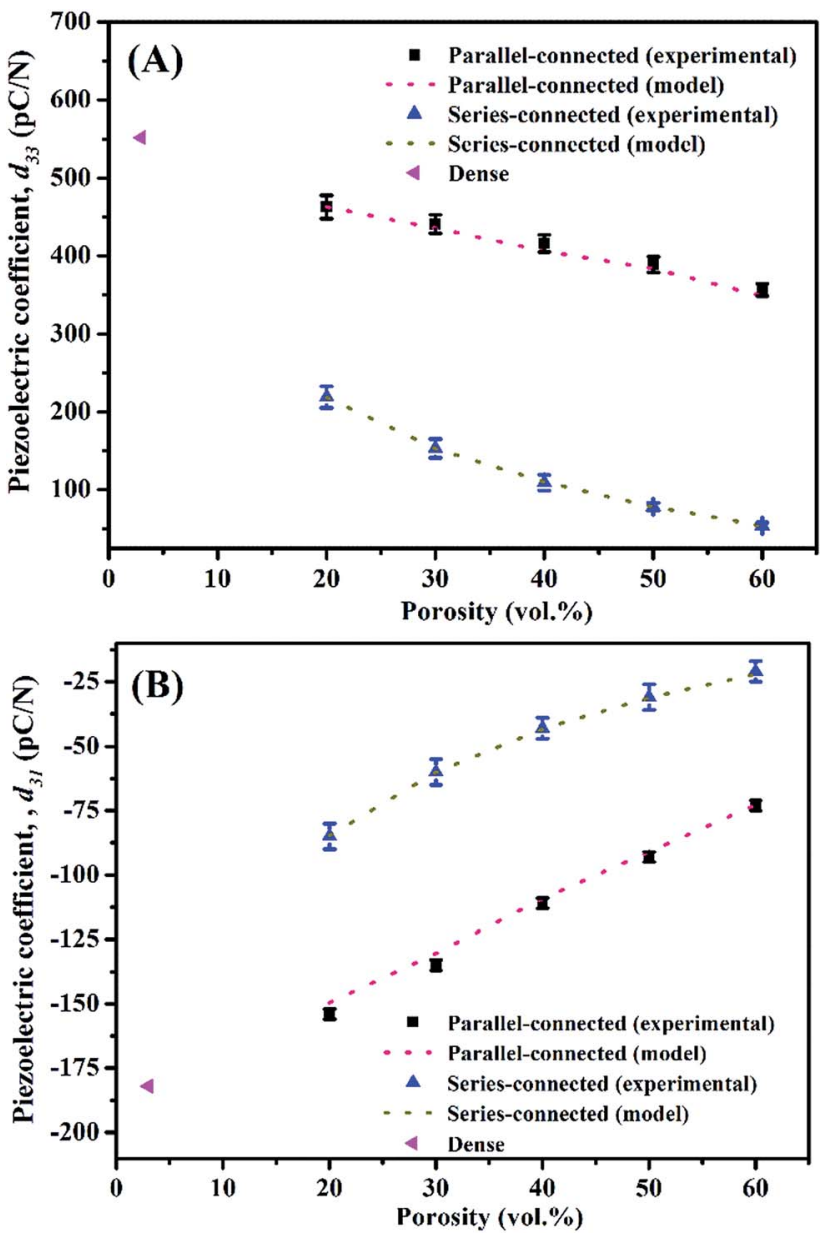

Fig. 6 (A) Longitudinal $\left(d_{33}\right)$ and (B) transverse $\left(d_{31}\right)$ piezoelectric coefficients of porous freeze-cast PZT ceramics with both experimental and modelling results as a function of the porosity. The dense material is also shown.

charge coefficients decreased almost linearly with an increase of the porosity from 20 to $60 \mathrm{vol} \%$, similar to the results from Piazza et al. ${ }^{37}$ who observed a decrease of $d_{33}$ in the porosity range from 4 vol\% to 45 vol\% for porous PZT using graphene as the pore-forming agent. This behaviour is due to the decrease of the polarisation of the material after the introduction of the porosity, as seen in the P-E loops in Fig. 4(B) and (D). Moreover, the presence of concentrated stress can exist in the irregular dendritic ceramic area on the surface of the aligned pores which can also be responsible for the decrease of piezoelectric coefficients compared with the dense PZT. The $d_{33}$ and $d_{31}$ of the parallel-connected porous PZT decreased by $16-35 \%$ and 15$58 \%$ respectively compared with the dense PZT, while there was a larger decrease in $d_{33}$ and $d_{31}$ of for the series-connected PZT (60-90\% and 53-88\%).

In addition, the parallel-connected porous PZT exhibited a longitudinal piezoelectric coefficient $d_{33}$ which was 3.0 to 4.7 times higher than that of the series-connected samples, shown in Fig. 6(A), while the $d_{31}$ was $\sim 2.5$ times higher than that of the series-modelled PZT, shown in Fig. 6(B). These results are consistent with the $P-E$ loops of Fig. 4 with a higher polarization 


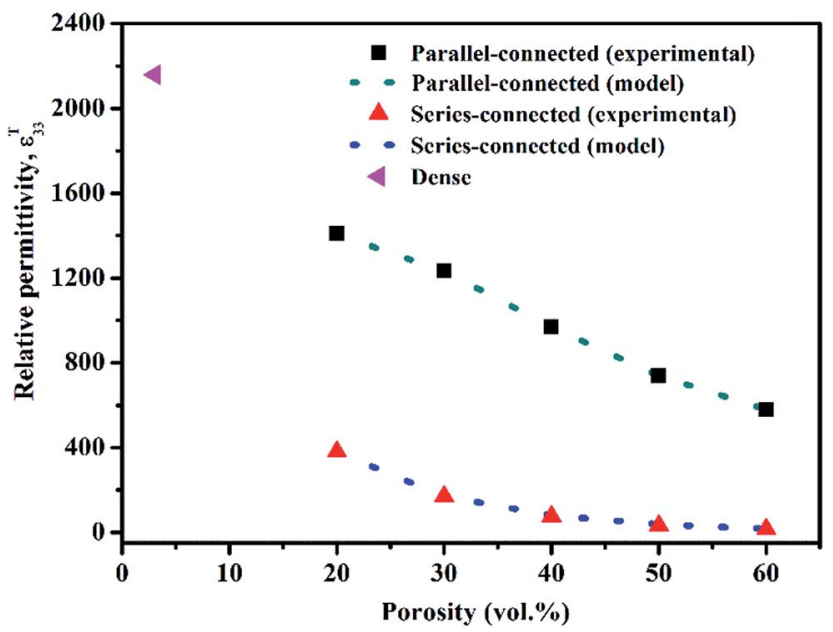

Fig. 7 Relative permittivity of porous freeze-cast PZT ceramics with both experimental and modelling results as a function of the porosity. The dense material is also shown.

in the parallel-connected PZT due the better interconnectivity of the PZT along the poling direction.

In the 2-2 connectivity composite, where the first phase is the active PZT piezoelectric ceramic, and the second phase is the passive pore channel (denoted as 'pc', henceforth), the piezoelectric coefficients for the series and parallel connection can be calculated by eqn (S1)-(S4). $\dot{\dagger}^{34}$ As can be seen from the SEM micrographs in Fig. 2, there are a number of ceramic bridges between the adjacent lamellar structures; therefore, in practice the aligned pore channel is a mixture of the PZT and air, rather than simply air. If we assume the piezoelectric coefficient and the elastic compliance of air are zero and infinite respectively, then the series and parallel equations can be simplified.

For series connection:

$$
\begin{gathered}
d_{33}=\frac{V^{\mathrm{PZT}} d_{33}^{\mathrm{PZT}}}{V^{\mathrm{PZT}}+V^{\mathrm{pc}} \times m} \\
d_{31}=\frac{V^{\mathrm{PZT}} d_{31}^{\mathrm{PZT}}}{\left(V^{\mathrm{pc}}+V^{\mathrm{pc}} \times m\right) \times\left(V^{\mathrm{PZT}}+V^{\mathrm{pc}} \times n\right)}
\end{gathered}
$$

For parallel connection:

$$
\begin{aligned}
& d_{33}=\frac{V^{\mathrm{PZT}} d_{33}^{\mathrm{PZT}}}{V^{\mathrm{PZT}}+V^{\mathrm{pc}} \times n} \\
& d_{31}=V^{\mathrm{PZT}} d_{31}^{\mathrm{PZT}}
\end{aligned}
$$

where $m$ is the ratio of the relative permittivity of the two phases $\left(\varepsilon_{33}^{\mathrm{PZT}} / \varepsilon_{33}^{\mathrm{pc}}\right)$, while $n$ is the ratio of the elastic compliances of the two phases $\left(s_{33}^{\mathrm{PZT}} / s_{33}^{\mathrm{pc}}\right)$. The calculated results are the dotted lines and the experimental values are the data points in Fig. 6. After fitting with the simplified equations from (7) to (10), the fitting parameter, $m$, increased slightly from 6.012 to 6.089 in the series-connected freeze-cast samples with an increase of porosity, while the fitting parameter, $n$, decreased slowly from
0.781 to 0.755 in the parallel-connected porous PZT (see Table $\mathrm{S} 1 \dagger$ ). Due to the small change of these two ratios, average values of $m=6.035$ and $n=0.763$ were utilised in the fitting curve, shown in Fig. 6. From the SEM micrographs in our previous research, the amount of the ceramic links in the aligned pore channels reduced when the porosity increased, accounting for the reduction of the permittivity $\varepsilon_{33}^{\mathrm{pc}}$ and the increase of the elastic compliance $s_{33}^{\mathrm{pc}}$ of the aligned pore channels. Moreover, it was noticed that compared with a ratio of $m \sim 6.035$ for the relative permittivity of the pore channel $\varepsilon_{33}^{\mathrm{pc}}$ than that of the ceramic wall $\varepsilon_{33}^{\mathrm{PZT}}$, the stiffness of the pore channel $1 / s_{33}^{\mathrm{pc}}$ was only $1.3(=1 / 0.763)$ times lower than that of the compliance of the lamellar ceramic wall $1 / s_{33}^{\mathrm{PZT}}$ in the $d_{33}$ of the parallelconnected and $d_{31}$ of the series-connected fitting values. As is known, nacre is a natural ceramic composite comprised of $95 \%$ calcium carbonate in the aragonite polymorph with $\sim 5 \%$ organic macromolecules sandwiched in between. However, its work of fracture is $\sim 3000$ times greater than monolithic ceramics. $^{38}$ The aligned porous structures via freeze-casting mimic the natural nacre structure due to the lamellar ceramic walls and the dendritic ceramic bridges were beneficial in stiffening the structure and limiting the torsion, ${ }^{39,40}$ which may be responsible for the reduced stiffness ratio in both the parallel and series-connected porous PZT ceramics.

Fig. 7 shows the relative permittivity of the dense and freezecast porous PZT measured at $1 \mathrm{kHz}$ and room temperature. The dielectric loss measured from 0.1 to $100 \mathrm{kHz}$ is also shown in Fig. $\mathrm{S} 3, \dagger$ with a similar loss for the ceramics across the porosity range. The relative permittivity decreased with increasing porosity in the range of $20-60 \mathrm{vol} \%$ due to the reduced volume fraction of the high permittivity material, which is consistent with previously reported data ${ }^{27}$ under different temperatures from 20 to $400{ }^{\circ} \mathrm{C}$, showing that the dielectric loss of both the porous and dense PZT remained almost constant below the Curie temperature. Moreover, the parallel-connected freeze-cast PZT exhibited 3.7-41.3 times higher relative permittivity than the series-connected PZT on increasing the porosity from 20 to $60 \mathrm{vol} \%$, which is due to the improved interconnection in the parallel-connected freeze-cast PZT ceramic. In addition, it is noted that the relative permittivity of the parallel-connected freeze-cast PZT decreased almost linearly with porosity, while there was a non-linear relationship in series-connected PZT, in particular a gentle decrease can be observed with porosity $>40$ vol\%. Lichtenecker's equation can be used to predict the dielectric function of a two-phase composite, ${ }^{41}$ given by eqn (11):

$$
\varepsilon_{\mathrm{L}}^{k}=v_{1} \varepsilon_{\mathrm{PZT}}{ }^{k}+v_{2} \varepsilon_{\mathrm{pc}}{ }^{k}
$$

where $\varepsilon_{\mathrm{L}}{ }^{k}$ is the composite relative permittivity, $v_{1}$ and $v_{2}$ are the volume fractions of PZT and pore channel in the porous PZT, respectively, $\varepsilon_{\mathrm{PZT}}{ }^{k}$ and $\varepsilon_{\mathrm{pc}}{ }^{k}$ are the relative permittivities of PZT and the pore channel, and $k$ varies within the range of $[-1,1]$, where $k=1$ for a fully parallel connection and $k=-1$ for a fully series connection. Fig. 7 shows the fitting curves of the dotted lines and Table S1† presents the corresponding fitting results. As shown in Fig. 7, both the parallel-connected and series- 
Table 1 Modelling parameters $(k)$ of relative permittivities of the freeze-cast porous PZT with both series and parallel connectivities

\begin{tabular}{lll}
\hline Porosity (\%) & Series-connected & Parallel-connected \\
\hline 20 & -0.016 & 0.511 \\
30 & -0.037 & 0.611 \\
40 & -0.038 & 0.632 \\
50 & -0.039 & 0.639 \\
60 & -0.043 & 0.692
\end{tabular}

connected freeze-cast PZT have a good fit with Lichtenecker's equation at all ranges of porosities. Theoretically, the parameter $k$ can be utilised to describe a transition from series anisotropy at $k=-1$ to parallel anisotropy at $k=1 .^{42}$ As shown in Table 1 , the $k$ in series-connected PZT decreased from -0.016 to -0.043 while in parallel-connected PZT the $k$ value increased from 0.813 to 0.905 with an increase of porosity, demonstrating that both types of freeze-cast ceramics possessed an increased degree of anisotropy with increasing porosity. Meanwhile, the reduced number of ceramic links in the aligned pore channels with high porosity can also be responsible for the higher degree of anisotropy of the freeze-cast ceramic.

Fig. 8 shows the piezoelectric generator figure of merit, $\mathrm{FoM}_{i j}$, calculated using eqn (1) at a constant stress as a function of porosity. For the longitudinal $d_{33}$ piezoelectric figure of merit, the parallel-connected PZT exhibited a higher value than the series-connected PZT. However, the parallel-connected PZT had a lower transverse $d_{31}$ piezoelectric figure of merit $\left(\mathrm{FoM}_{31}\right)$ in the whole porosity range. In the series-connected PZT, both longitudinal $\mathrm{FoM}_{33}$ and transverse $\mathrm{FoM}_{31}$ piezoelectric figures of merit increase with increasing porosity and were higher than those of the dense material. The increase in the figures of merit with porosity in Fig. 8 results from the large decrease in relative permittivity with increasing porosity in Fig. 7. However, in the parallel-connected PZT, the transverse FoM $_{31}$ figure of merit

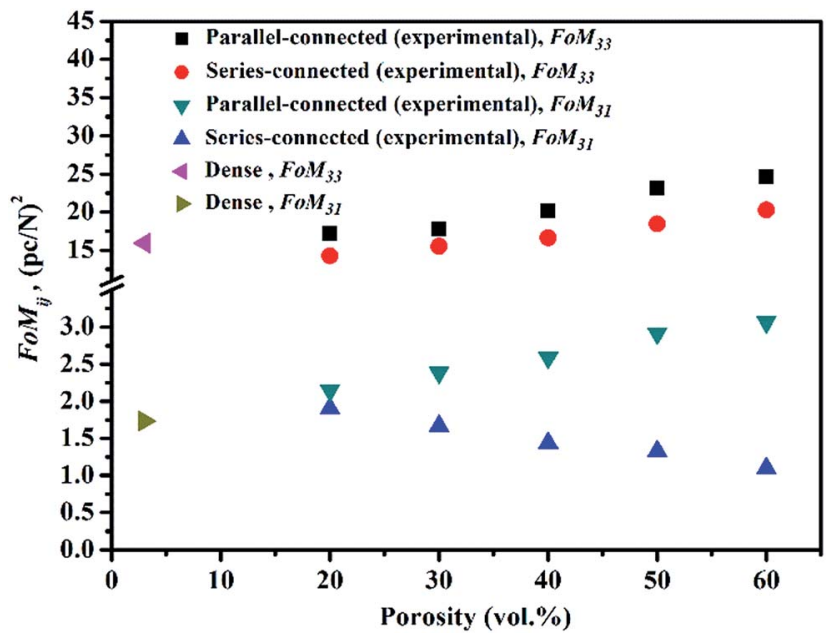

Fig. 8 Piezoelectric FoM $\mathrm{F}_{33}$ and $\mathrm{FoM}_{31}$ generator figure of merit at a constant stress of porous freeze-cast PZT ceramics as a function of porosity. The dense material is also shown. decreased with increasing porosity and was lower than that of the dense material when the porosity $>40 \mathrm{vol} \%$. This is due to the more rapid decrease of the $d_{31}$ (Fig. 6(B)) compared to the permittivity (Fig. 7) with porosity for this orientation. A further increase in the porosity level beyond 60 vol\% can result in a decrease of the piezoelectric figure of merit since $d_{33}$ falls rapidly at higher porosity; ${ }^{43}$ in addition it is of no benefit to the mechanical strength, as shown in Fig. S1. $\dagger$

\subsection{Pyroelectric properties}

Fig. 9(A) and (B) show the pyroelectric coefficients and pyroelectric figure of merit $\left(F_{\mathrm{E}}^{\prime}\right)$ of porous freeze-cast PZT ceramics. Theoretical pyroelectric formulations for the series and parallel connections are given by eqn (S5) and (S6) $\uparrow$ respectively. After simplifying these equations in a similar way to the above piezoelectric coefficients, eqn (12) and (13) are obtained as below.

For the series connection:

$$
p=\frac{V^{\mathrm{PZT}} p^{\mathrm{PZT}}}{V^{\mathrm{PZT}}+V^{\mathrm{pc}} \times m}
$$

For the parallel connection:

$$
p=V^{\mathrm{PZT}} p^{\mathrm{PZT}}
$$

Fig. 9(A) shows that the pyroelectric coefficient decreased in both types of freeze-cast PZT ceramics with increasing porosity, and is lower than that of the dense PZT. The parallel-connected PZT had a higher pyroelectric coefficient than the seriesconnected PZT at the same porosity, e.g. $3.0 \mu \mathrm{C} \mathrm{m}^{-2} \mathrm{~K}^{-1}$ and $4.0 \mu \mathrm{C} \mathrm{m}^{-2} \mathrm{~K}^{-1}$ for the series-connected PZT and parallelconnected PZT, respectively, at the $40 \mathrm{vol} \%$ porosity. As seen in Fig. 4, the decreased polarization in the porous samples results in the decreased pyroelectric coefficient $(p)$ due to the relationship between the pyroelectric coefficient and polarization where $p=\mathrm{d} P / \mathrm{d} T$, and $\mathrm{d} P$ and $\mathrm{d} T$ are the change in polarization and temperature respectively. The measured specific heat capacities of the porous PZT were 260, 227, 201, 163, and $140 \mathrm{~J} \mathrm{~kg}^{-1} \mathrm{~K}^{-1}$ at room temperature when the porosity increased from 20 to 60 vol\%, as shown in Fig. 9(B). The $F^{\prime}{ }_{E}$ figures of merit for thermal harvesting (eqn (2)) are shown in Fig. 9(C). Although the pyroelectric coefficient of the parallel-connected PZT decreased by $\sim 49 \%$, see Fig. 9 (A), the corresponding figure of merit $F_{\mathrm{E}}^{\prime}=p^{2} /\left(\varepsilon_{0} \varepsilon_{33}^{T} \times\left(C_{\mathrm{E}}\right)^{2}\right)$ increased by $\sim 405 \%$ when the porosity increased from 20 to 60 vol\% due to the combination of the reduced permittivity (Fig. 7) and specific heat capacity (Fig. 9(B)). The same trends were also found in the series-connected PZT with a larger decrease in the pyroelectric coefficient and lower increase in $F_{\mathrm{E}}^{\prime}$ which were $\sim 76 \%$ and $\sim 151 \%$, respectively. The modelling results demonstrate a good fit with the experimental data.

In addition to potential energy harvesting applications, there are other important pyroelectric detection figures of merit to describe the performance of a material as a pyroelectric sensor. ${ }^{44}$ These include the current responsivity, $F_{\mathrm{I}}=p / C_{\mathrm{E}}$, voltage responsivity, $F_{\mathrm{V}}=p /\left(C_{\mathrm{E}} \varepsilon_{33}{ }^{T} \varepsilon_{0}\right)$ and signal to noise figure 

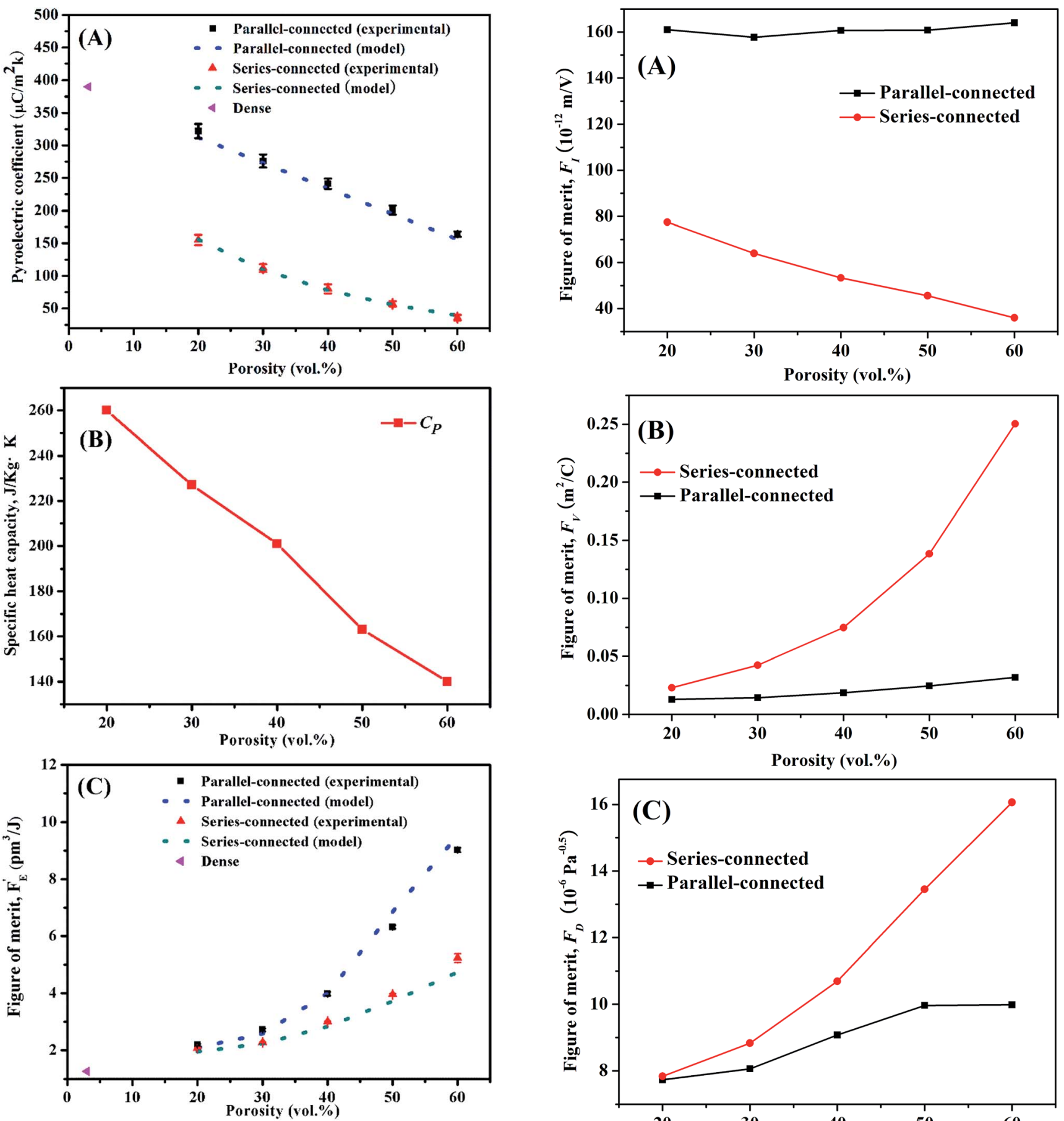

Fig. 9 (A) Pyroelectric coefficients, (B) specific heat capacity and (C) pyroelectric figure of merit of porous freeze-cast PZT ceramics with both experimental and modelling results as a function of porosity. The dense material is also shown.

of merit, $F_{\mathrm{D}}=p /\left(C_{\mathrm{E}}\left(\varepsilon_{33}{ }^{T} \varepsilon_{0} \tan \delta\right)^{0.5}\right)$, where $C_{\mathrm{E}}$ is the heat capacity. The current responsivity, $F_{\mathrm{I}}$, remains constant with increasing porosity for the parallel connected material since the decrease in pyroelectric coefficient is similar to the decrease in heat capacity, see Fig. 10(A). For the series connected material there is a decrease in $F_{\mathrm{I}}$, due to the low pyroelectric coefficient in this direction (Fig. 9(A)). The voltage responsivity, $F_{\mathrm{V}}$ in

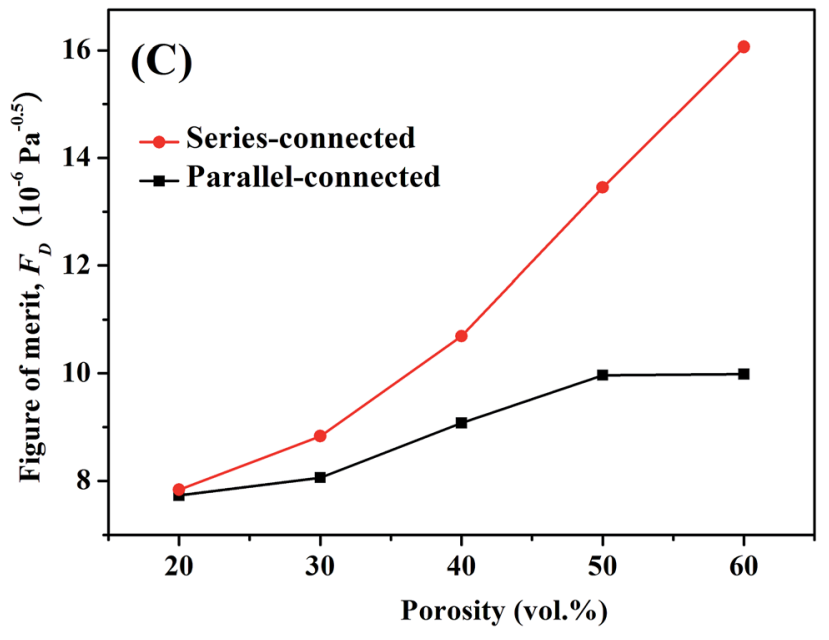

Fig. 10 (A) Figures of merit for the pyroelectric detectors. (A) Current responsivity $F_{\mathrm{l}},(\mathrm{B})$ voltage responsivity $F_{\mathrm{V}}$ and $(\mathrm{C}) F_{\mathrm{D}}$.

Fig. 10(B), increases with increasing porosity in both directions due to the decrease in heat capacity and permittivity. The $F_{\mathrm{V}}$ is larger for the series connected materials due to the lower permittivity, see Fig. 7. Finally, $F_{\mathrm{D}}$ increases with increasing porosity, Fig. 10(C), in both directions due to the reduced specific heat and permittivity; changes in loss are relatively small. Interestingly, while the series connection is advantageous for the $F_{\mathrm{V}}$ 
and $F_{\mathrm{D}}$ detection figures of merit, the parallel connection is highest for the $F_{\mathrm{E}}$ harvesting figure of merit, see Fig. $9(\mathrm{C})$; this is due to its $p^{2}$ dependency (see eqn (2)) and higher pyroelectric coefficient in this direction (Fig. 9(A)).

\subsection{Pyroelectric energy harvesting demonstrator}

As a consequence of the reduced permittivity (Fig. 7) and volume specific heat capacity (Fig. 9(B)) it can be seen that the $F^{\prime}{ }_{\text {E }}$ pyroelectric harvesting figure of merit increased with an increase of the porosity (as in Fig. 9(C)) despite the decreasing pyroelectric coefficients. Furthermore, in order to demonstrate the potential of porous materials with improved energy harvesting figures of merit a demonstrator system for harvesting thermal fluctuations was developed. Fig. 11(A)-(D) show the pyroelectric energy harvesting system, a schematic of the full bridge rectifier circuit with the AC-DC rectifier and storage capacitor, and the voltage level of the capacitor along with the generated electric energy/energy density with time during charging the capacitor as the pyroelectric element is subjected to temperature oscillations. In this work, an electrometer was used to measure the voltage of the charging capacitor generated by the porous PZT with parallel-connected porosity due to the improved figures of merit with increasing porosity, as seen in Fig. 10(C). A Philips HP3631/01 IR lamp, with a power of $175 \mathrm{~W}$, was employed as the heat source for heating and cooling the PZT elements. In particular, in a thermal energy harvesting application, the AC signals generated by the energy harvesting materials (both dense and the aligned porous PZT ceramics) must be rectified and stored in a capacitor. A full wave rectifier circuit similar to our previous report ${ }^{45}$ is shown in Fig. 11(B), where the pyroelectric element is considered as a charge source with a shunt capacitor and resistor. The magnitude of the current/voltage varies with the porosity level of the piezoelectric element at the same temperature fluctuation.

The measured forward voltage drop of the rectifier circuit was $2 \mathrm{~V}$ and during the charging process, the energy is $C V^{2} / 2$, where $C$ and $V$ are the capacitance and the voltage of the capacitor, respectively. It can be clearly seen that on increasing the level of aligned porosity from $20 \mathrm{vol} \%$ to $60 \mathrm{vol} \%$, the parallel-connected porous PZT produced an increasing peak voltage from 8.3 to $15.2 \mathrm{~V}$ after $3600 \mathrm{~s}$ (Fig. 11(C)) with a corresponding increase of energy from $34 \mu \mathrm{J}$ to $116 \mu \mathrm{J}$ (Fig. 11(D)) and energy density from 490 to $1653 \mu \mathrm{J} \mathrm{cm}^{-3}$ (inset in Fig. 11(D)). The dense PZT had the lowest voltage of $7.8 \mathrm{~V}$, energy $(31 \mu \mathrm{J})$ and energy density $\left(446 \mu \mathrm{J} \mathrm{cm}^{-3}\right)$. Both the voltage and the energy density increased with an increase of the porosity level, which is entirely consistent with the improvement in the pyroelectric figure of merit shown in Fig. 10(B). A maximum voltage of $15.2 \mathrm{~V}$ was obtained in the storage capacitor utilising parallel-connected PZT with the highest porosity of $60 \mathrm{vol} \%$ which demonstrated the fastest charging speed to obtain a stable voltage level of the capacitor, and the corresponding maximum energy and energy density $\left(1653 \mu \mathrm{J} \mathrm{cm}^{-3}\right)$ were $374 \%$ higher than those of the dense PZT $\left(446 \mu \mathrm{J} \mathrm{cm}^{-3}\right)$.

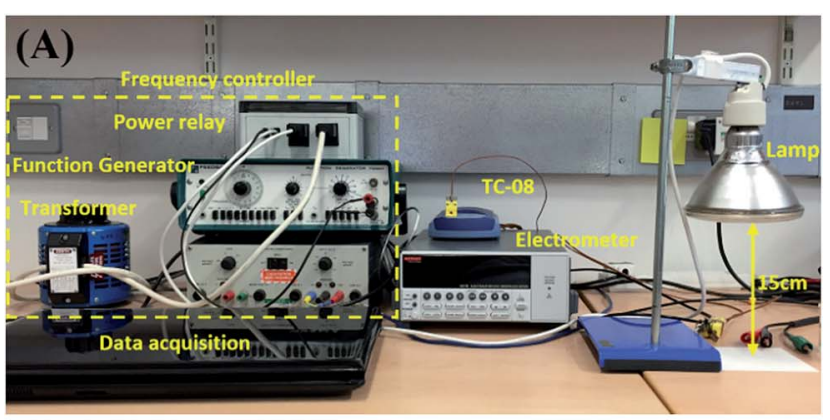

(B)
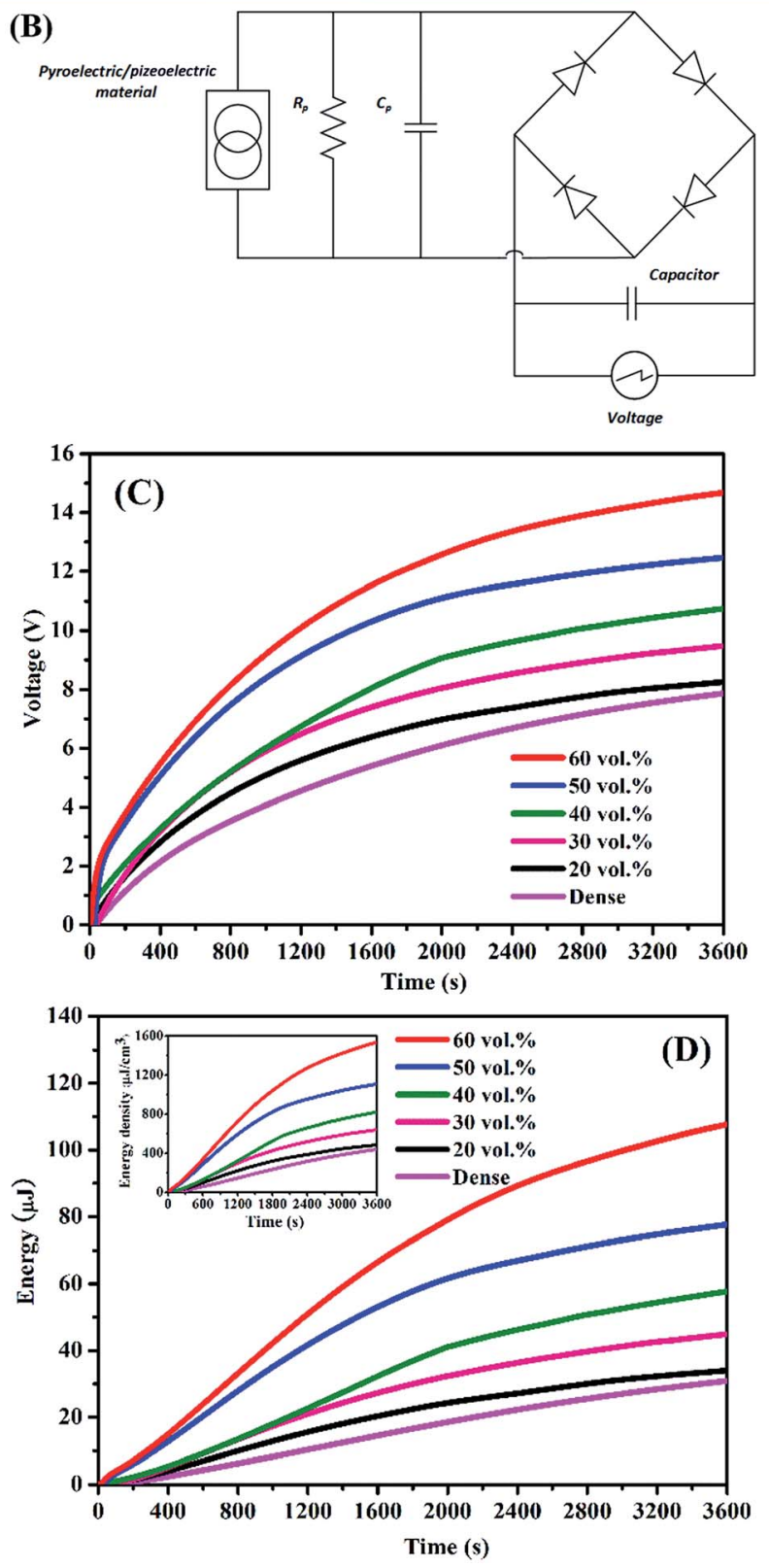

Fig. 11 (A) The test setup platform of the pyroelectric energy harvester. (B) A schematic diagram of a pyroelectric power generator with circuitry. (C) Charging voltage vs. time plots of the dense and parallel-connected porous PZT with various porosities ranging from 20 to $60 \mathrm{vol} \%$ and the dense material. (D) Charging energy of the capacitor, the inset shows the charging energy density. 


\section{Conclusions}

This paper has demonstrated the significant benefits of using porosity to enhance the piezoelectric and pyroelectric properties of materials for energy harvesting. Novel 2-2 connectivity PZT ceramics with aligned pore channels have been prepared by freeze-casting water-based PZT suspensions, with porosity levels ranging from 20 to $60 \mathrm{vol} \%$. The unidirectional lamellar microstructure and the strong lamellar bonding originating from the ceramic bridges between the lamellar walls were shown to be beneficial to achieve a higher compressive strength compared with the conventional porous PZT with uniformly distributed porosity. The change in remnant polarization and coercive field with porosity level is described and explained with modelling of the electric field distribution within the two-phase microstructure. The parallel-connected porous PZT exhibited a $300 \%$ to $470 \%$ higher longitudinal piezoelectric coefficient $d_{33}$ and $\sim 250 \%$ times higher transverse piezoelectric coefficient $d_{31}$ than the series-connected PZT in the complete porosity range of 20 to $60 \mathrm{vol} \%$. As a result of the reduced permittivity and reduced specific heat capacity, the aligned porous PZT demonstrated excellent piezoelectric and pyroelectric figures of merit, compared with the dense counterpart. The modelling results utilising the coupling of the ratio of the dielectric constant and elastic compliance between the ceramic wall and pore channel showed good agreement with the experimental results. To demonstrate the improved performance of the aligned porous materials a pyroelectric energy harvesting device was constructed and clearly demonstrated that the fastest charging speed and maximum energy density were obtained when utilising the highest porosity of $60 \mathrm{vol} \%$ for a parallelconnected PZT. The measured energy density was $374 \%$ higher than that of the dense PZT and demonstrates that porous materials are highly attractive materials for energy harvesting applications.

\section{Acknowledgements}

Dr Y. Zhang would like to acknowledge the European Commission's Marie Skłodowska-Curie Actions (MSCA), through the Marie Skłodowska-Curie Individual Fellowships (IF-EF) (H2020MSCA-IF-2015-EF-703950-HEAPPs) under Horizon 2020. Prof. C. R. Bowen would like to acknowledge funding from the European Research Council under the European Union's Seventh Framework Programme (FP/2007-2013)/ERC Grant Agreement no. 320963 on Novel Energy Materials, Engineering Science and Integrated Systems (NEMESIS). Prof. D. Zhang would like to acknowledge the support from State Key Laboratory of Powder Metallurgy, Central South University. The authors are also thankful for the experimental supports from Ms Xi Yuan and Ms Fengdan Xue, and the helpful advice and inspiring discussions with Dr Hamideh Khanbareh and Mr Marcin Krasny.

\section{References}

1 C. R. Bowen, H. A. Kim, P. M. Weaver and S. Dunn, Energy Environ. Sci., 2014, 7, 25-44.
2 J. T. C. Bowen, E. LeBoulbar, D. Zabek, A. Chauhan and R. Vaish, Energy Environ. Sci., 2014, 7, 25-44.

3 D. Guyomar, Y. Jayet, L. Petit, E. Lefeuvre, T. Monnier, C. Richard and M. Lallart, Sens. Actuators, A, 2007, 138, 151-160.

4 T. L. Ling Bing Kong, H. H. Hng, F. Boey, T. Zhang and S. Li, Waste energy harvesting, Springer, Berlin Heidelberg, 2014.

5 J. Nowotny, T. Hoshino, J. Dodson, A. J. Atanacio, M. Ionescu, V. Peterson, K. E. Prince, M. Yamawaki, T. Bak, W. Sigmund, T. N. Veziroglu and M. A. Alim, Int. J. Hydrogen Energy, 2016, 41, 12812-12825.

6 A. O. Korotkevich, Z. S. Galochkina, O. Lavrova and E. A. Coutsias, Renewable Energy, 2015, 81, 804-807.

7 S. P. Beeby, M. J. Tudor and N. M. White, Meas. Sci. Technol., 2006, 17, R175.

8 E. Arroyo and A. Badel, Sens. Actuators, A, 2011, 171, 266-273. 9 E. O'Riordan, D. Galayko, P. Basset and E. Blokhina, Sens. Actuators, A, 2016, 247, 379-388.

10 M. Sobocinski, M. Leinonen, J. Juuti and H. Jantunen, J. Eur. Ceram. Soc., 2011, 31, 789-794.

11 J. Kim and J.-H. Koh, J. Eur. Ceram. Soc., 2015, 35, 3819-3825.

12 F. Y. Lee, A. Navid and L. Pilon, Appl. Therm. Eng., 2012, 37, 30-37.

13 F. Delorme, M. Bah, F. Schoenstein, F. Jean, M. Zouaoui Jabli, I. Monot-Laffez and F. Giovannelli, Mater. Lett., 2016, 162, 24-27.

14 C.-H. Chiu, W.-I. Liang, C.-W. Huang, J.-Y. Chen, Y.-Y. Liu, J.-Y. Li, C.-L. Hsin, Y.-H. Chu and W.-W. Wu, Nano Energy, 2015, 17, 72-81.

15 G. Cha, Y. Jia and Y. S. Ju, 2012 IEEE 25th International Conference on Micro Electro Mechanical Systems (MEMS), 2012, 1241-1244, DOI: 10.1109/memsys.2012.6170414.

16 A. Navid, D. Vanderpool, A. Bah and L. Pilon, Int. J. Heat Mass Transfer, 2010, 53, 4060-4070.

17 C. R. Bowen, J. Taylor, E. LeBoulbar, D. Zabek and V. Topolov, Mater. Lett., 2015, 138, 243-246.

18 T. Rödig, A. Schönecker and G. Gerlach, J. Am. Ceram. Soc., 2010, 93, 901-912.

19 R. V. K. Mangalam, J. C. Agar, A. R. Damodaran, J. Karthik and L. W. Martin, ACS Appl. Mater. Interfaces, 2013, 5, 13235-13241.

20 P. Talemi, M. Delaigue, P. Murphy and M. Fabretto, ACS Appl. Mater. Interfaces, 2015, 7, 8465-8471.

21 V. Y. Topolov, S. V. Glushanin and C. R. Bowen, Adv. Appl. Ceram., 2005, 104, 300-305.

22 N. Ashcon and P. Laurent, Smart Mater. Struct., 2011, 20, 025012.

23 Y. Wang, W. Zhong and P. Zhang, J. Appl. Phys., 1993, 74, 521-524.

24 H. H. S. Chang and Z. Huang, Smart Mater. Struct., 2010, 19, 065018.

25 V. Y. Topolov, A. V. Krivoruchko, C. R. Bowen and A. A. Panich, Ferroelectrics, 2010, 400, 410-416.

26 J. Roscow, Y. Zhang, J. Taylor and C. Bowen, Eur. Phys. J.: Spec. Top., 2015, 224, 2949-2966.

27 Y. Zhang, Y. Bao, D. Zhang and C. R. Bowen, J. Am. Ceram. Soc., 2015, 98, 2980-2983. 
28 Y. Zhang, K. C. Zhou, J. Zeng and D. Zhang, Adv. Appl. Ceram., 2013, 112, 405-411.

29 Y. Zhang, L. Chen, J. Zeng, K. C. Zhou and D. Zhang, Mater. Sci. Eng., C, 2014, 39, 143-149.

30 S. Deville, E. Saiz and A. P. Tomsia, Acta Mater., 2007, 55, 1965-1974.

31 European Standard EN 623-2:1993 Advanced technical ceramics - Monolithic ceramics - General and textural properties - Part 2 - Determination of density and porosity. 32 R. L. Byer and C. B. Roundy, IEEE Trans. Sonics Ultrason., 1972, 19, 333-338.

33 C. Hong, X. Zhang, J. Han, J. Du and W. Zhang, Mater. Chem. Phys., 2010, 119, 359-362.

34 R. E. Newnham, D. P. Skinner and L. E. Cross, Mater. Res. Bull., 1978, 13, 525-536.

35 L. Padurariu, L. P. Curecheriu and L. Mitoseriu, Acta Mater., 2016, 103, 724-734.

36 R. W. C. Lewis, A. C. E. Dent, R. Stevens and C. R. Bowen, Smart Mater. Struct., 2011, 20, 085002.
37 D. Piazza, C. Capiani and C. Galassi, J. Eur. Ceram. Soc., 2005, 25, 3075-3078.

38 A. P. Jackson, J. F. V. Vincent and R. M. Turner, Proc. R. Soc. London, Ser. B, 1988, 234, 415-440.

39 G. W. Bailey, S. McKeman, R. L. Price, S. D. Walck, P. M. Charest and R. Gauvin, Structural Details as Clues to Understanding Nacre Formation, Springer-Verlag, New York, NY, 2000.

40 F. Bouville, E. Maire, S. Meille, B. Van de Moortèle, A. J. Stevenson and S. Deville, Nat. Mater., 2014, 13, 508-514. 41 A. V. Goncharenko, V. Z. Lozovski and E. F. Venger, Opt. Commun., 2000, 174, 19-32.

42 K. Lichtenecker, Phys. Z., 1926, 27, 115-158.

43 J. I. Roscow, J. Taylor and C. R. Bowen, Ferroelectrics, 2016, 498, 40-46.

44 R. W. Whatmore, Rep. Prog. Phys., 1986, 49, 1335-1386.

45 M. Y. Xie, D. Zabek, C. R. Bowen, M. Abdelmageed and M. Arafa, Smart Mater. Struct., 2016, 25, 125023. 